\title{
Procesamiento de Arvejas (Pisum sativum L.). Parte 1: Modelado de la Cinética de Secado por Capa Delgada de Arveja, Variedades Obonuco Andina y Sureña
}

\author{
Darío C. Pantoja(1), Oswaldo Osorio(2), Diego F. Mejía(2) y Henry A. Váquiro(3) \\ (1) Ingeniería Agroindustrial. Universidad de Nariño. Facultad de Ingeniería Agroindustrial. Pasto. \\ Colombia. (2) Universidad de Nariño. Facultad de Ingeniería Agroindustrial. Grupo de Apoyo a la \\ Investigación y Desarrollo Agroalimentario (GAIDA) y Tecnologías Emergentes en Agroindustria (TEA). \\ Pasto. Colombia. \\ (3) Universidad del Tolima. Facultad de Ingeniería Agronómica. Grupo de investigación CEDAGRITOL, \\ Ibagué. Colombia. (e-mail: camilopantojaes@gmail.com; osorio_oswaldo@hotmail.com; \\ diegomejiaespana@gmail.com; hvaquiro@gmail.com
}

Recibido Ago. 25, 2015; Aceptado Oct. 7, 2015; Versión final Nov. 11, 2015, Publicado Feb. 2016

\begin{abstract}
Resumen
En este estudio se realizó el secado de arveja variedades Obonuco Andina y Sureña a temperaturas entre 45 y $60^{\circ} \mathrm{C}$ con velocidad de aire de $1.5 \mathrm{~m} / \mathrm{s}$. El incremento de la temperatura redujo el tiempo de proceso en ambas variedades. El modelo de Verma describió satisfactoriamente el proceso en términos del coeficiente de determinación ajustado, el error medio relativo y los intervalos de confianza para los parámetros del modelo. La difusividad efectiva $\left(D_{\text {eff }}\right)$ y la energía de activación $\left(E_{a}\right)$ se calcularon empleando la solución analítica de la segunda Ley de Fick para geometría esférica y resistencia externa despreciable. La difusividad efectiva $D_{\text {eff }}$ se incrementó con el aumento de la temperatura, fluctuando entre $1.649 \times 10^{-10}$ y $2.585 \times 10^{-10} \mathrm{~m}^{2} / \mathrm{s}$ para variedad Obonuco Andina y entre $1.752 \times 10^{-10}$ y $2.586 \times 10^{-10}$ $\mathrm{m}^{2} / \mathrm{s}$ para la variedad Sureña. La energía de activación $E_{a}$ para la variedad Obonuco Andina fue de 26.87 $\mathrm{kJ} / \mathrm{mol}$ y para la variedad Sureña fue de $25.29 \mathrm{~kJ} / \mathrm{mol}$.
\end{abstract}

\section{Processing of Peas (Pisum sativum L.). Part 1: Modelling of the Thin Layer Drying Kinetic of Pea, Varieties Obonuco Andina and Sureña}

\begin{abstract}
In this study the drying of peas, varieties Obonuco Andina and Sureña, at temperatures between 45 and $60^{\circ} \mathrm{C}$ with air velocity of $1.5 \mathrm{~m} / \mathrm{s}$ was carried out. The temperature increase reduced the process time for both varieties. Verma's model properly described the process in terms of the adjusted coefficient of determination, mean relative error and confidence intervals for the model parameters. The effective diffusivity $\left(D_{\text {eff }}\right)$ and activation energy $\left(E_{a}\right)$ were calculated using the analytical solution of the second Fick's Law for spherical geometry and negligible external resistance. The effective diffusivity $D_{\text {eff }}$ increased when the temperature was increased, ranging between $1.649 \times 10^{-10}$ and $2.585 \times 10^{-10} \mathrm{~m}^{2} / \mathrm{s}$ to variety Obonuco Andina and between $1.752 \times 10^{-10}$ and $2.586 \times 10^{-10} \mathrm{~m}^{2} / \mathrm{s}$ for variety Sureña. The activation energy $\mathrm{E}_{\mathrm{a}}$ for the variety Obonuco Andina was $26.87 \mathrm{~kJ} / \mathrm{mol}$ and for the variety Sureña was $25.29 \mathrm{~kJ} / \mathrm{mol}$.
\end{abstract}

Keywords: peas; drying; modelling; effective diffusivity; activation energy 


\section{INTRODUCCION}

La arveja (Pisum sativum L.) es una leguminosa de gran importancia en Colombia y en especial en el Sur de Nariño, cuyo consumo se da en fresco, deshidratado, enlatado, entre otras presentaciones que manifiestan su potencialidad en cuanto a industrialización se refiere. La arveja deshidratada posee gran popularidad puesto que ofrece ventajas como su prolongada vida útil en estado seco, palatividad y conveniencia de transporte y manipulación. De igual manera al ser un producto tan perecedero en estado fresco (actividad de agua de 0.98 a $18^{\circ} \mathrm{C}$ ), un inadecuado manejo del material vegetal representaría pérdidas económicas para productores, comercializadores y consumidores, es aquí donde el secado del material vegetal puede dar una alternativa de solución a esta problemática (Garg et al., 2014).

Durante el secado puede presentarse una transferencia de humedad por difusión del interior hasta la superficie del alimento. Posteriormente, en la interface solido-gas la humedad es retirada de la superficie por convección. El objetivo principal del proceso es disminuir el contenido de humedad y la actividad de agua del producto para evitar la consecuente degradación bioquímica y microbiológica durante el almacenamiento y posterior transformación o consumo; esto posibilita la reducción de gastos por pérdidas y el aumento de la calidad del producto final (Fonseca et al., 2007; Michalewicx et al., 2011). Actualmente se han llevado a cabo estudios del comportamiento del secado de diferentes productos hortofrutícolas como maracuyá (Bezerra et al., 2015), mango (García et al., 2015), limón (Puente et al., 2013), nopal (Martinez et al., 2010), anacardo (Machado et al., 2010), fríjol (Doymaz, 2005), soja (Torrez y Giner, 2014), pimiento rojo (Vega et al., 2005) y arveja (Pardeshi et al., 2009; Doymaz y Kocayigit, 2011; Priyadarshini et al., 2013; Sahin et al., 2013; Zielinska et al., 2013). Estos estudios resaltan la importancia que tiene el estudio de la cinética de secado y el modelado de datos experimentales para efectos de control y optimización de procesos de secado.

Partiendo de la complejidad de la cinética de secado al involucrar transferencia de masa y calor en régimen transitorio, se requieren de modelos confiables que describan el comportamiento del producto tratado durante el proceso. Existen dos tipos de modelos de capa delgada ampliamente usados: modelos teóricos desarrollados a partir de principios físicos y que implican soluciones analíticas de ecuaciones de difusión tomando en cuenta ciertas consideraciones; y modelos empíricos, obtenidos a partir de análisis matemático o estadístico de los datos de proceso, partiendo de ecuaciones más sencillas pero con aplicabilidad al control y estudio de ciertas variables del secado (Kaleta y Górnicki, 2010). Entre los modelos utilizados se destacan los modelos de Weibull, Page, Henderson y Pabis, Dos Términos Exponencial, Logarítmico, Verma y Midilli (Menges y Ertekin, 2006; Pardeshi et al., 2009).

Cabe resaltar que diversos estudios han concluido que el mecanismo de transporte predominante en el secado de alimentos está relacionado con fenómenos de transferencia internos por difusión líquida. Por ello es crucial evaluar propiedades como la difusividad efectiva y la energía de activación, los cuales se determinan a partir de modelos basados en la Segunda Ley de Fick. La determinación de dichas propiedades de transporte podría ser importante para el estudio del proceso con el propósito de obtener productos de buena calidad y para implementar procesos energéticamente eficientes y amigables con el medio ambiente (Bezerra et al., 2015). De la misma manera, la difusividad efectiva corresponde a una propiedad con significado físico por medio de la cual se puede caracterizar un producto en comparación con otros, teniendo en cuenta la capacidad de un material heterogéneo para transportar humedad. Por otro lado, la energía de activación y el factor pre exponencial definen el cambio del coeficiente de difusividad con respecto a la temperatura de secado. El conocimiento de dicha variación es necesario en la simulación del proceso de secado a partir de fenómenos de transporte de materia y calor. A su vez, la simulación del proceso puede utilizarse como herramienta para establecer condiciones óptimas de operación que involucren criterios relacionados con la calidad del producto, la eficiencia energética y, por tanto, con la rentabilidad del proceso (Mujumdar, 2007).

Existen muchas referencias relacionadas con el comportamiento de productos agrícolas durante el secado; sin embargo no existen estudios relacionados con el modelado de secado de las variedades de arveja producidas en el departamento de Nariño y comercializadas a gran parte de Colombia. Es así como el objetivo de este trabajo fue determinar la cinética de secado por convección de dos variedades de arveja, producidas en el sur de Colombia y aplicar modelos matemáticos de capa delgada.

\section{MATERIALES Y METODOS}

\section{Obtención y adecuación de la muestra}

Las muestras de arveja variedad Obonuco Andina y Sureña fueron suministradas por el proyecto financiado por el Sistema General de Regalías "Evaluación de la Aptitud de Nuevas Líneas de Arveja (Pisum sativum L.) Para el Procesamiento Agroindustrial Actualmente Aptas Agronómicamente en el Departamento de 
Nariño". Ambas variedades fueron producidas en la finca LOPE del Centro Nacional de Aprendizaje SENA regional Nariño. Inicialmente se realizó el desgranado, selección y clasificación (según tamaño y forma, descartando granos excesivamente frescos, deformes o con presencia de plagas). Posteriormente las muestras fueron sometidas a condiciones constantes de temperatura y humedad relativa $\left(20^{\circ} \mathrm{C}, 60 \% \mathrm{HR}\right)$ con el fin de garantizar uniformidad en las propiedades de las arvejas. Para ello el material vegetal se dispuso en una bandeja y esta a su vez en una nevera de poliestireno expandido con cloruro de calcio en su interior. La exposición de la muestra de arveja se prolongó por un período de $12 \mathrm{~h}$ previas al secado (Senadeera, 2008).

\section{Equipos y procedimiento}

Se usó una cámara de secado tipo incubadora (IN-601, Lab Incubator, Gemmy Industrial Corp, Taiwan) que consta de tres unidades básicas, un ventilador cuya velocidad de aire generada tenía una magnitud constante de $1.5 \mathrm{~m} / \mathrm{s}$, un sistema de calentamiento por resistencias eléctricas en el interior del equipo provisto de un controlador digital de temperatura, y una cámara de secado en la cual se podían disponer cuatro bandejas de $30 \mathrm{~cm} \times 30 \mathrm{~cm}$. El equipo se encendió 30 min antes de iniciar las sesiones con el fin de alcanzar las condiciones estables de trabajo y posteriormente se cargó con $360 \mathrm{~g}$ de arveja por cada experimento.

\section{Construcción de las curvas de secado}

El proceso de secado de Pisum sativum L. var. Obonuco Andina y var. Sureña se evaluó por triplicado a temperaturas de 45, 50, 55 y $60 \stackrel{\circ}{\circ}$. Se midió el contenido humedad empleando un Determinador Electrónico de Humedad (Versión 1.1, Kern DBS, KERN \& Sohn GmbH, Alemania); se tomaron muestras de $2 \mathrm{~g}$ de arveja cada 10 min durante las primeras $2 \mathrm{~h}$ y posteriormente cada 20 min hasta finalizar el proceso. Para la finalización del proceso se consideró la obtención de un peso constante, asumido como una variación inferior al $1 \%$ en cuatro lecturas de humedad consecutivas. Previamente a la medición del contenido de humedad, las muestras fueron dispuestas en un desecador con cloruro de calcio con el fin de evitar la transferencia de humedad del material vegetal y el ambiente (Zielinska et al., 2013).

\section{Modelado matemático}

Los datos obtenidos se transformaron a humedad en base seca y se calcularon los respectivos promedios. Posteriormente se determinó la razón de humedad (MR) dada por la ecuación 1 con el fin ajustar a 8 modelos matemáticos descritos en la tabla 1, estos modelos han sido reportados por diversos estudios en productos agrícolas entre los que se destaca el estudio llevado a cabo por Priyadarshini et al. (2013).

$\mathrm{MR}=\frac{\mathrm{M}_{\mathrm{t}}-\mathrm{M}_{\mathrm{e}}}{\mathrm{M}_{\mathrm{o}}-\mathrm{M}_{\mathrm{e}}}$

donde $M_{t}$ corresponde a la humedad ( $\mathrm{kg} / \mathrm{kg}$ base seca) registrada en el tiempo $\mathrm{t}(\mathrm{s}), \mathrm{Me}_{\mathrm{e}}$ es la humedad de equilibrio ( $\mathrm{kg} / \mathrm{kg}$ b.s.) asumida como el valor registrado a tiempos prolongados de proceso (Guiné y Barroca, 2011) y $M_{0}$ es el contenido de humedad inicial ( $\mathrm{kg} / \mathrm{kg}$ b.s.). Los índices de ajuste correspondieron al coeficiente de determinación ajustado (ecuación 2) y error medio relativo (ecuación 3).

$\mathrm{R}_{\text {adj }}^{2}=1-\left(1-\mathrm{R}^{2}\right) \frac{\mathrm{N}-1}{\mathrm{~N}-\mathrm{m}-1}$

Donde $R^{2}$ adj corresponde al coeficiente de determinación ajustado, $R^{2}$ corresponde al coeficiente de determinación, $\mathrm{m}$ es el número de parámetros de regresión y $\mathrm{N}$ es el número de observaciones. Según Villa-Velez et al. (2015) el parámetro $\mathrm{R}^{2}$ adj corresponde a un indicador apropiado de modelado puesto que considera la variación de los datos experimentales por medio del coeficiente de determinación, y el número de parámetros por cada modelo matemático.

$\mathrm{MRE}=\frac{100}{N} \sum_{i=1}^{N}\left(\frac{\left|M R_{\exp }-M R_{\text {pre }}\right|}{M R_{\exp }}\right)$

Donde MRE es el error medio relativo (\%), $\mathrm{N}$ es el número de observaciones, MRexp es el valor i-ésimo de la razón de humedad experimental y MRpre es el valor i-ésimo de la razón de humedad predicha

En la tabla 1, MR es la razón de humedad, t es el tiempo, y las variables $a, b, c, g, k, n, k_{0}$ y $k_{1}$ son constantes de cada modelo según corresponda. Los parámetros de cada modelo fueron estimados usando un método de regresión no lineal de la herramienta estadística Matlab® R2011b (The MathWorks Inc., 
Natick, MA, USA) a través de la función "nlinfit" Los intervalos de confianza se calcularon con la función "nlparci" para valorar si los parámetros son estadísticamente significativos en el modelo ( Kaymak, 2002; Eim, 2012).

Tabla 1. Modelos matemáticos aplicados a las curvas de secado

\begin{tabular}{|l|l|}
\hline Modelo & Ecuación \\
\hline Weibull & $\mathrm{MR}=\exp (-\mathrm{t} / \mathrm{b})$ \\
\hline Page & $\mathrm{MR}=\exp \left(-\mathrm{kt} \mathrm{n}^{\mathrm{n}}\right)$ \\
\hline Henderson y Pabis & $\mathrm{MR}=\mathrm{a} \exp (-\mathrm{kt})$ \\
\hline Dos Términos Exponencial & $\mathrm{MR}=\mathrm{a} \exp (-\mathrm{kt})+(1-\mathrm{a}) \exp (-\mathrm{kat})$ \\
\hline Logarítmico & $\mathrm{MR}=\mathrm{a} \exp (-\mathrm{kt})+\mathrm{c}$ \\
\hline Verma & $\mathrm{MR}=\mathrm{a} \exp (-\mathrm{kt})+(1-\mathrm{a}) \exp (-\mathrm{gt})$ \\
\hline Midilli & $\mathrm{MR}=\mathrm{a} \exp \left(-\mathrm{kt} \mathrm{t}^{\mathrm{n}}\right)+\mathrm{bt}$ \\
\hline Dos Términos & $\mathrm{MR}=\mathrm{a} \exp \left(-\mathrm{k}_{0} \mathrm{t}\right)+\mathrm{b} \exp \left(-\mathrm{k}_{1} \mathrm{t}\right)$ \\
\hline
\end{tabular}

Calculo de difusividad efectiva y energía de activación

Dado que la forma de los granos de arveja se asemeja a la de una esfera, el coeficiente de difusividad puede ser calculado por medio de la solución analítica de la segunda Ley de Fick para cuerpos esféricos (ecuación 4), al considerar que el material es homogéneo e isótropo, que el proceso es isotérmico, que el efecto de la resistencia externa y la contracción sobre la transferencia de materia son despreciables (Simal, 1996).

$M R=\frac{6}{\pi^{2}} \sum_{n=1}^{\infty} \frac{1}{n^{2}} \exp \left(-n^{2} \pi^{2} \frac{D_{\text {eff }} \mathrm{t}}{r^{2}}\right)$

Donde MR es la razón de humedad, $n$ es el número de término, Deff es la difusividad efectiva $\left(\mathrm{m}^{2} / \mathrm{s}\right)$ y $\mathrm{r}$ es el radio de la esfera $(\mathrm{m})$.

El cálculo de la energía de activación se realizó considerando la dependencia de la difusividad efectiva con la temperatura (Váquiro, 2009) por medio de una ecuación tipo Arrhenius (ecuación 5).

$D_{e f f}=D_{o} \exp \left[-\frac{E_{a}}{R(T+273.15)}\right]$

En esta ecuación, $D_{\text {eff }}$ es la difusividad efectiva $\left(\mathrm{m}^{2} / \mathrm{s}\right)$, Do es el factor pre-exponencial de la ecuación Arrhenius $\left(\mathrm{m}^{2} / \mathrm{s}\right), \mathrm{E}_{\mathrm{a}}$ es la energía de activación $(\mathrm{kJ} / \mathrm{mol}), \mathrm{R}$ es la constante universal de los gases (0.00831 $\mathrm{kJ} / \mathrm{molK}$ ) y $\mathrm{T}$ es la temperatura en ${ }^{\circ} \mathrm{C}$.

La ecuación 3 fue resuelta considerando los 15 primeros términos de la serie. Los parámetros $D_{\circ}$ y $E_{a}$ fueron identificados al minimizar la suma de los errores absolutos entre los datos experimentales y calculados, mediante la herramienta Solver de MS Excelß.

\section{RESULTADOS Y DISCUSIÓN}

En las figuras 1 y 2 se puede observar la variación de la humedad respecto al tiempo de secado a 45, 50, 55 y $60^{\circ} \mathrm{C}$. Al igual que lo obtenido por Jovanovic (2013), se evidencia que un incremento en la temperatura disminuye el contenido de humedad a un tiempo dado, es decir, que la velocidad transferencia de materia se incrementa con la temperatura.

Tal y como lo manifiesta Vega et al. (2005), se evidencia un efecto de la temperatura sobre el proceso, al incrementarse la temperatura de secado, se incrementa la velocidad del mismo. Similares resultados reportan Martínez (2012) en semillas de uva; Torregroza et al. (2014) en yuca, Djendoubi et al., (2012) en pera, Simal et al. (1996) en arveja; Hatamipour y Mowla (2003) en guisantes y maíz. Se asume que la difusión es el principal mecanismo físico que define el movimiento de humedad dentro del grano de arveja, fenómeno recalcado por Pardeshi et al. (2009) y por Sahin et al. (2013), quienes afirman además que los productos agrícolas normalmente describen el periodo de velocidad decreciente durante su secado. 


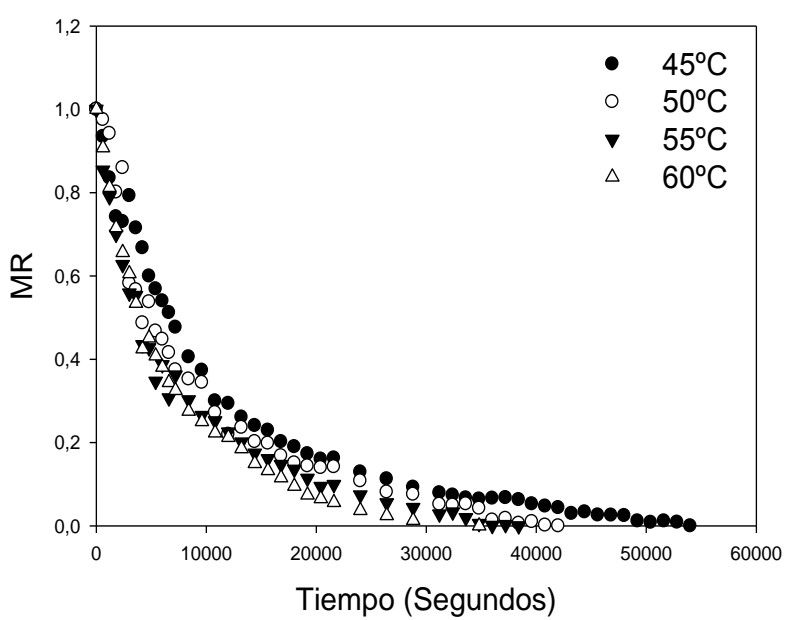

Fig. 1: Curvas de secado para arveja variedad Obonuco Andina.

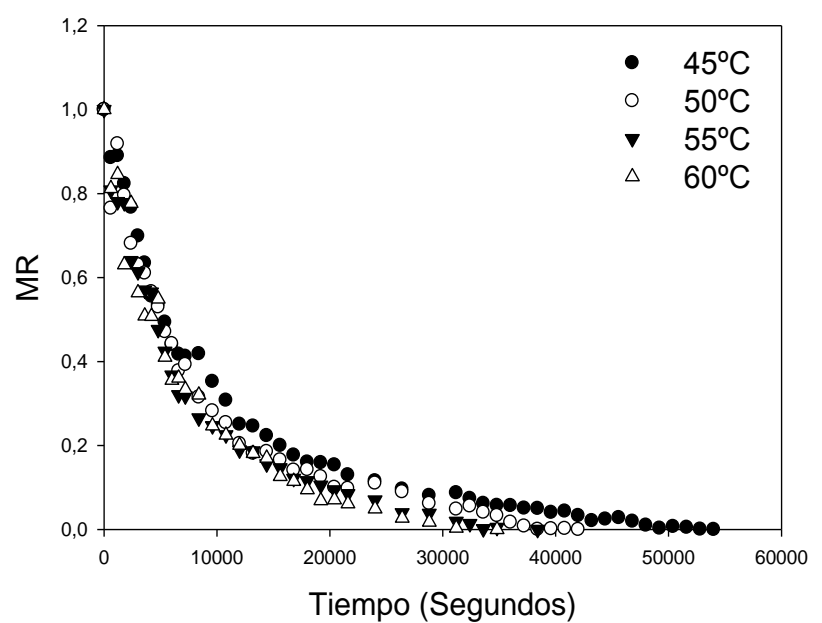

Fig. 2: Curvas de secado para arveja variedad Sureña.

Tabla 2. Resultados estadísticos de los 8 modelos evaluados a diferentes temperaturas por cada variedad de arveja.

\begin{tabular}{|c|c|c|c|c|c|}
\hline \multirow{2}{*}{$T\left({ }^{\circ} \mathrm{C}\right)$} & \multirow{2}{*}{ Modelo } & \multicolumn{2}{|c|}{ Var. Obonuco Andina } & \multicolumn{2}{|c|}{ Var. Sureña } \\
\hline & & $R^{2} \mathrm{adj}$ & $\operatorname{MRE}(\%)$ & $R^{2} \mathrm{adj}$ & MRE (\%) \\
\hline \multirow[t]{8}{*}{45} & Weibull & 0.994 & 2.46 & 0.991 & 2.92 \\
\hline & Page & 0.994 & 1.45 & 0.994 & 1.70 \\
\hline & Henderson y Pabis & 0.992 & 2.11 & 0.989 & 2.59 \\
\hline & Dos términos exponencial & 0.994 & 1.41 & 0.995 & 1.69 \\
\hline & Logarítmico & 0.993 & 1.75 & 0.991 & 2.24 \\
\hline & Verma & 0.994 & 1.32 & 0.995 & 1.14 \\
\hline & Midilli & 0.994 & 1.44 & 0.994 & 1.69 \\
\hline & Dos términos & 0.994 & 1.34 & 0.996 & 1.35 \\
\hline \multirow[t]{8}{*}{50} & Weibull & 0.978 & 4.36 & 0.987 & 3.84 \\
\hline & Page & 0.986 & 2.03 & 0.995 & 1.45 \\
\hline & Henderson y Pabis & 0.973 & 3.50 & 0.984 & 2.39 \\
\hline & Dos términos exponencial & 0.985 & 2.66 & 0.993 & 2.23 \\
\hline & Logarítmico & 0.977 & 3.07 & 0.986 & 2.17 \\
\hline & Verma & 0.986 & 1.75 & 0.994 & 1.62 \\
\hline & Midilli & 0.985 & 1.99 & 0.995 & 1.45 \\
\hline & Dos términos & 0.987 & 1.75 & 0.993 & 1.64 \\
\hline \multirow[t]{8}{*}{55} & Weibull & 0.982 & 4.34 & 0.987 & 3.23 \\
\hline & Page & 0.992 & 1.73 & 0.990 & 2.00 \\
\hline & Henderson y Pabis & 0.978 & 3.22 & 0.986 & 2.62 \\
\hline & Dos términos exponencial & 0.991 & 2.41 & 0.989 & 2.14 \\
\hline & Logarítmico & 0.981 & 3.05 & 0.986 & 2.57 \\
\hline & Verma & 0.994 & 1.46 & 0.989 & 1.97 \\
\hline & Midilli & 0.993 & 1.54 & 0.989 & 1.93 \\
\hline & Dos términos & 0.994 & 1.47 & 0.989 & 2.04 \\
\hline \multirow[t]{8}{*}{60} & Weibull & 0.991 & 2.71 & 0.981 & 3.33 \\
\hline & Page & 0.996 & 1.23 & 0.983 & 2.46 \\
\hline & Henderson y Pabis & 0.990 & 2.18 & 0.980 & 2.97 \\
\hline & Dos términos exponencial & 0.997 & 1.06 & 0.983 & 2.35 \\
\hline & Logarítmico & 0.991 & 2.01 & 0.980 & 3.00 \\
\hline & Verma & 0.997 & 1.06 & 0.983 & 2.34 \\
\hline & Midilli & 0.996 & 1.05 & 0.982 & 2.24 \\
\hline & Dos términos & 0.997 & 1.08 & 0.982 & 2.44 \\
\hline
\end{tabular}


En la tabla 2 se puede observar que los valores de $R^{2}$ adj y MRE presentaron valores que indican la bondad de ajuste de los datos experimentales a los modelos empíricos evaluados. El coeficiente de determinación ajustado obtuvo valores mayores e iguales a 0.97, aspecto destacado por Doymaz y Kocayigit (2011). Del mismo modo se obtuvo MRE inferiores al 10\%, resultado que para Corrêa et al. (2011) demuestra un buen ajuste a los datos experimentales.

Los intervalos de confianza permitieron inferir que cada uno de los términos de los modelos Weibull, Page, Henderson y Pabis y Verma, tuvieron significancia estadística en la predicción del parámetro MR en todos los factores estudiados (temperatura y variedad de arveja). Además de contar con altos valores de $R^{2}{ }_{a d j} y$ bajos para MRE, los rangos comprendidos entre los respectivos límites superiores e inferiores de los intervalos de confianza no incluyen el cero y por ende resultan ser ecuaciones apropiadas para describir la relación entre el tiempo y la humedad adimensional.

El modelo Weibull es reportado como un modelo adecuado para describir el secado de mango (Corzo et al., 2010) maíz amarillo, soja, gandul, soja roja y arroz (Hui, 2006). Por otra parte el modelo Page es ampliamente reportado en arveja, Doymaz y Kocayigit (2011) obtuvieron buenos resultados para el modelo en el secado de arveja con un equipo de convección forzada a temperaturas entre 55 y $70^{\circ} \mathrm{C}$; también Garg et al. (2014) resaltan el modelo nombrado como el adecuado para describir el secado de arveja a $70^{\circ} \mathrm{C}$ en un equipo convencional. Por otra parte Mechlouch et al. (2014) y Priyadarshini et al. (2013) reportan buenos resultados para el modelo de Page en el secado de arveja con microondas. El modelo Henderson y Pabis es resaltado por Joykumar y Pandey (2012) en el secado convencional de batata; Dutta y Baruah (2014) en el secado de té negro y Martinez et al. (2010) en el secado de nopal por lecho fluidizado.

El modelo Verma mostró ser el más apropiado para representar el secado de las variedades de arveja estudiadas puesto que además presentar mayores valores de $R^{2}$ adj y menores valores de MRE, los términos de la ecuación, para cada temperatura estudiada, son estadísticamente significativos. Las figuras 3 y 4 presentan el ajuste entre los valores experimentales y calculados por medio del modelo Verma para la variedad Obonuco Andina y Sureña respectivamente. La variedad Obonuco Andina presentó coeficiente de determinación entre los rangos 0.988 a 0.997 , y errores comprendidos entre 1.06 y $1.75 \%$. Por su parte la variedad Sureña presentó valores entre 0.983 a 0.996 y entre 1.14 a $2.37 \%$ respectivamente. Pese que Pardeshi et al. (2009) consolidaron el modelo Aproximación a la difusión como el que mejor se ajustó al secado de arveja, los autores resaltan el modelo Verma por obtener MRE inferiores al $10 \%$. Igualmente se han obtenido resultados adecuados para el modelo Verma en estudios llevados a cabo por Hacihafizoğlu et al. (2008) en arroz, Rafiee et al. (2009) en soja y Bispo et al., (2015) en piña.

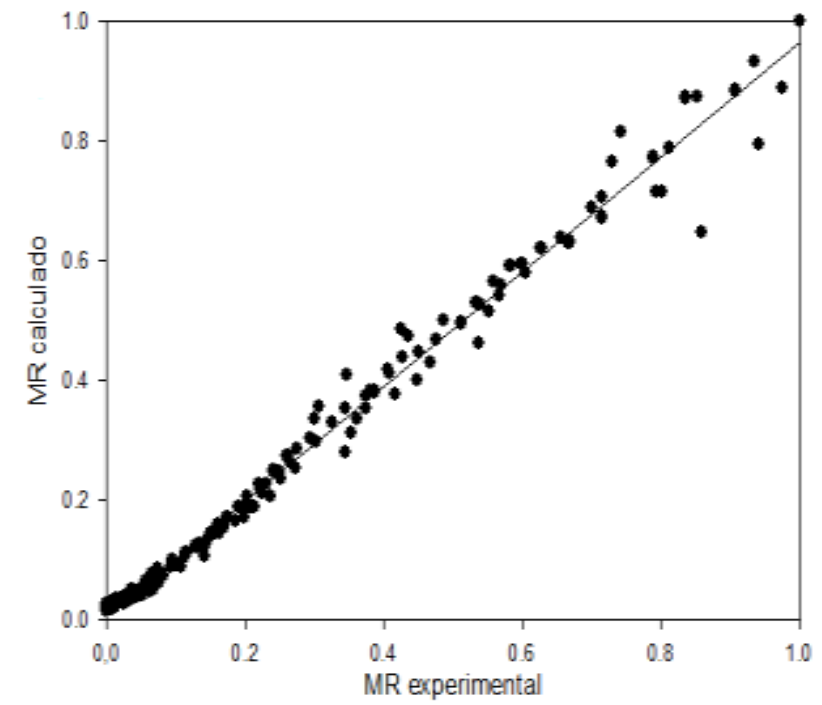

Fig. 3: MR experimental y calculada por medio del modelo de Verma para la arveja variedad Obonuco Andina.

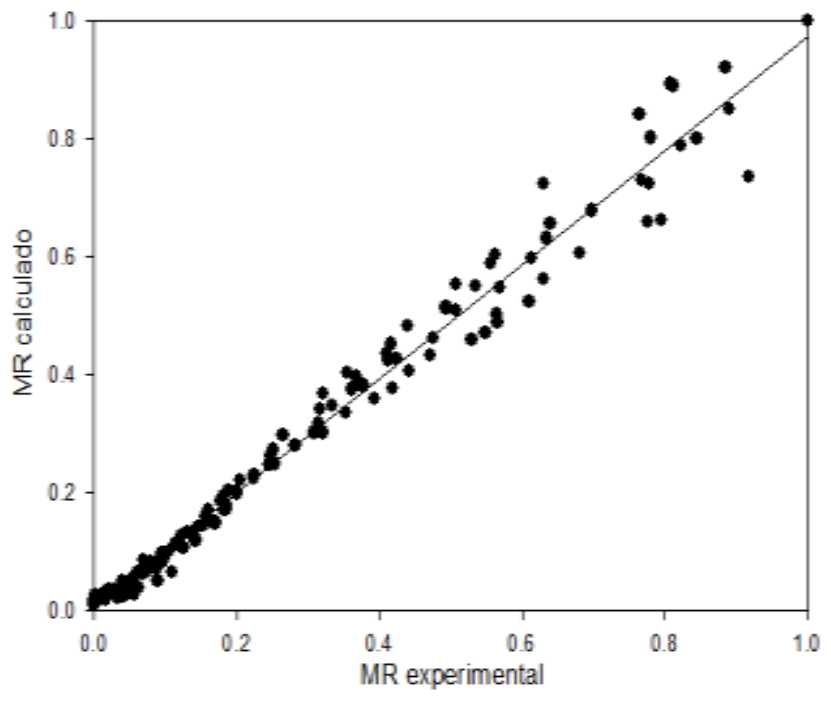

Fig. 4: MR experimental y calculada por medio del modelo de Verma para la arveja variedad Sureña

En las tablas 3 y 5 se presentan los valores de los parámetros del modelo Verma para cada temperatura evaluada, así como el coeficiente $R^{2}$ adj y MRE tanto para la variedad Obonuco Andina como para la variedad Sureña. Por otro lado, en las tablas 4 y 6 se presentan los intervalos de confianza (95\%) para cada uno de los parámetros del modelo. 
El modelo Verma y en general, los modelos estudiados están construidos a partir del ajuste estadístico de los parámetros de las ecuaciones correspondientes, a datos experimentales de humedad adimensional. Es así como, al igual que en otros estudios, se reporta que las constantes de dichos modelos, no presentan una dependencia definida con la variación de la temperatura. Por esta razón se considera que estas aproximaciones matemáticas no proveen una interpretación de los fenómenos que gobiernan el fenómeno ocurrido durante la remoción de agua del material vegetal de la variedad Obonuco Andina y Sureña (Rodriguez, 2013). No obstante se han realizado cierta cantidad de investigaciones buscando determinar modelos empíricos que describan el comportamiento de alimentos, los autores dan relevancia a esta clase de modelos puesto que permiten establecer una relación directa entre el contenido medio de humedad del producto y el tiempo de proceso (Perea, 2011).

Tabla 3. Parámetros del modelo Verma para arveja variedad Obonuco Andina

\begin{tabular}{|c|c|c|c|c|c|}
\hline \multirow{2}{*}{$T\left({ }^{\circ} \mathrm{C}\right)$} & \multicolumn{3}{|c|}{ Parámetros } & \multirow{2}{*}{$R^{2}$ adj } & \multirow{2}{*}{ MRE (\%) } \\
\hline & a (adimensional) & $k(1 / s)$ & $g(1 / s)$ & & \\
\hline 45 & $5.246 \times 10^{-1}$ & $1.651 \times 10^{-4}$ & $6.011 \times 10^{-5}$ & 0.994 & 1.32 \\
\hline 50 & $4.459 \times 10^{-1}$ & $3.615 \times 10^{-4}$ & $7.627 \times 10^{-5}$ & 0.988 & 1.75 \\
\hline 55 & $4.036 \times 10^{-1}$ & $4.492 \times 10^{-4}$ & $8.699 \times 10^{-5}$ & 0.994 & 1.46 \\
\hline 60 & $3.063 \times 10^{-1}$ & $4.469 \times 10^{-4}$ & $1.088 \times 10^{-4}$ & 0.997 & 1.06 \\
\hline
\end{tabular}

Tabla 4. Intervalos de confianza (95\%) de los parámetros del modelo de Verma para arveja variedad Obonuco Andina

\begin{tabular}{|c|c|c|c|}
\hline$T\left({ }^{\circ} \mathrm{C}\right)$ & a (adimensional) & $k(1 / \mathrm{s})$ & $g(1 / \mathrm{s})$ \\
\hline 45 & $1.762 \times 10^{-1}, 9.916 \times 10^{-5}$ & $3.877 \times 10^{-5}, 8.730 \times 10^{-1}$ & $2.311 \times 10^{-4}, 8.144 \times 10^{-5}$ \\
\hline 50 & $2.691 \times 10^{-1}, 2.074 \times 10^{-4}$ & $5.752 \times 10^{-5}, 6.228 \times 10^{-1}$ & $5.155 \times 10^{-4}, 9.503 \times 10^{-5}$ \\
\hline 55 & $2.953 \times 10^{-1}, 3.069 \times 10^{-4}$ & $7.402 \times 10^{-5}, 5.120 \times 10^{-1}$ & $5.915 \times 10^{-4}, 9.995 \times 10^{-5}$ \\
\hline 60 & $1.746 \times 10^{-1}, 2.630 \times 10^{-4}$ & $9.430 \times 10^{-5}, 4.381 \times 10^{-1}$ & $6.308 \times 10^{-4}, 1.232 \times 10^{-4}$ \\
\hline
\end{tabular}

Tabla 5. Parámetros del modelo Verma para arveja variedad Sureña

\begin{tabular}{|c|c|c|c|c|c|}
\hline \multirow{2}{*}{$\mathrm{T}\left({ }^{\circ} \mathrm{C}\right)$} & \multicolumn{3}{|c|}{ Parámetros } & \multirow{2}{*}{$R^{2} a d j$} & \multirow{2}{*}{ MRE (\%) } \\
\hline & a (adimensional) & $k(1 / s)$ & $g(1 / s)$ & & \\
\hline 45 & $5.297 \times 10^{-1}$ & $2.048 \times 10^{-4}$ & $6.068 \times 10^{-5}$ & 0.996 & 1.14 \\
\hline 50 & $2.546 \times 10^{-1}$ & $1.004 \times 10^{-3}$ & $1.016 \times 10^{-4}$ & 0.994 & 1.62 \\
\hline 55 & $3.839 \times 10^{-1}$ & $3.510 \times 10^{-4}$ & $9.466 \times 10^{-5}$ & 0.989 & 1.97 \\
\hline 60 & $2.038 \times 10^{-1}$ & $5.356 \times 10^{-4}$ & $1.164 \times 10^{-4}$ & 0.983 & 2.37 \\
\hline
\end{tabular}

Tabla 6. Intervalos de confianza (95\%) de los parámetros del modelo de Verma para arveja variedad Sureña

\begin{tabular}{|c|c|c|c|}
\hline$T\left({ }^{\circ} \mathrm{C}\right)$ & a (adimensional) & $k(1 / \mathrm{s})$ & $g(1 / \mathrm{s})$ \\
\hline 45 & $3.522 \times 10^{-1}, 1.523 \times 10^{-4}$ & $4.732 \times 10^{-5}, 7.071 \times 10^{-1}$ & $2.572 \times 10^{-4}, 7.403 \times 10^{-5}$ \\
\hline 50 & $2.008 \times 10^{-1}, 5.518 \times 10^{-4}$ & $9.307 \times 10^{-5}, 3.084 \times 10^{-1}$ & $1.456 \times 10^{-3}, 1.101 \times 10^{-4}$ \\
\hline 55 & $9.299 \times 10^{-2}, 1.254 \times 10^{-4}$ & $6.587 \times 10^{-5}, 6.747 \times 10^{-1}$ & $5.765 \times 10^{-4}, 1.235 \times 10^{-4}$ \\
\hline 60 & $-5.440 \times 10^{-2},-1.999 \times 10^{-4}$ & $8.766 \times 10^{-5}, 4.621 \times 10^{-1}$ & $1.270 \times 10^{-3}, 1.451 \times 10^{-4}$ \\
\hline
\end{tabular}

\section{Difusividad efectiva (Deff)}

Los valores de difusividad efectiva obtenidos se muestran en la figura 5 . Respectivamente para las temperaturas de secado de 45, 50, 55 y $60^{\circ} \mathrm{C}$, la variedad de arveja Obonuco Andina obtuvo valores de $1.649 \times 10^{-10}, 1.890 \times 10^{-10}, 2.224 \times 10^{-10}$ y $2.585 \times 10^{-10} \mathrm{~m}^{2} / \mathrm{s}$. Por otro lado la variedad Sureña obtuvo valores en su orden, de $1.752 \times 10^{-10}, 2.087 \times 10^{-10}, 2.399 \times 10^{-10}$ y $2.586 \times 10^{-10} \mathrm{~m}^{2} / \mathrm{s}$.

El coeficiente $\mathrm{R}^{2}$ adj obtenido entre los valores experimentales y los valores calculados por medio de la ecuación de Fick variaron entre 0.969 y 0.989 para la variedad Obonuco Andina, y entre 0.970 y 0.9859 para la variedad Sureña. Como lo afirmó Thuwapanichayanan et al. (2011), la difusividad efectiva aumenta junto con la temperatura puesto que se disminuye la resistencia interna del sólido para la migración de humedad libre, conforme se eleva la temperatura, la porosidad del grano es mayor y se facilita la migración de agua por área superficial.

Se observa que los valores de difusividad efectiva, a cada temperatura de secado, variaron tanto para la variedad Obonuco Andina como para la variedad Sureña. Este fenómeno es mencionado por Hernández et 
al. (2011) quienes afirman que son variaciones normales debido a la anisotropía de los alimentos, igualmente Barroca y Guiné (2013) en la cinética de secado de dos variedades de banano, afirman que ciertas fluctuaciones en los aspectos cinéticos de secado, se deben a variaciones en el contenido de humedad inicial y en general a la variación experimental. Igual relevancia toma lo manifestado por Chinenye et al. (2010) en un estudio de secado de cacao, quienes atribuyen ciertas variaciones a los aspectos genéticos de los cultivares evaluados.

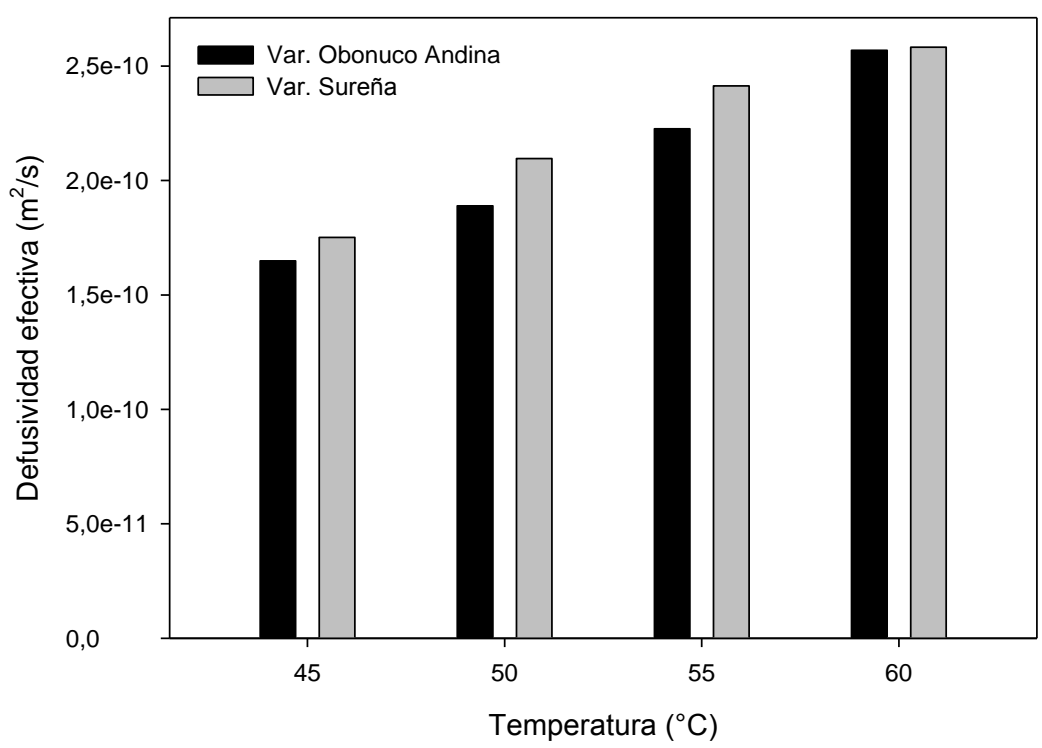

Fig. 5: Difusividad efectiva para la arveja variedad Obonuco Andina y Sureña según la temperatura de secado.

Los valores obtenidos se acercan a lo reportado por Pardeshi et al. (2009) al establecer que la difusividad efectiva de la arveja varía entre $3.95 \times 10^{-10} \mathrm{~m}^{2} / \mathrm{s}$ y $6.23 \times 10^{-10} \mathrm{~m}^{2} / \mathrm{s}$ en un rango comprendido entre los 55 y $75^{\circ} \mathrm{C}$; del mismo modo Doymaz y Kocayigit (2011) reportaron resultados comprendidos entre $8.059 \times 10^{-11} \mathrm{y}$ $1.973 \times 10^{-10} \mathrm{~m}^{2} / \mathrm{s}$, bajo temperaturas de secado entre 55 y $70 \stackrel{\circ}{\circ}$. No obstante los resultados obtenidos en la presente investigación se ubican en el rango reportado para alimentos mencionado por Puente et al. (2013) comprendido entre el orden de $10^{-12}$ y $10^{-9} \mathrm{~m}^{2} / \mathrm{s}$.

\section{Energía de activación (Ea)}

La energía de activación calculada para la variedad de arveja Obonuco Andina fue de $26.867 \mathrm{~kJ} / \mathrm{mol}$ (intervalo de confianza 95\%: 23.16; 30.58), mientras que para la variedad Sureña fue de $25.291 \mathrm{~kJ} / \mathrm{mol}$ (intervalo de confianza 95\%: $11.5 ; 39.08$ ). Estas magnitudes representan la energía requerida para iniciar la difusión de humedad de las dos variedades de arveja durante el secado. Los valores corresponden a lo reportado en otras investigaciones como la realizada por Torregroza et al. (2014) quienes afirman que la energía de activación de los alimentos fluctúa entre 12.7 y $110 \mathrm{~kJ} / \mathrm{mol}$. Concretamente para arveja, Pardeshi et al. (2009) reportan un valor de $22.48 \mathrm{~kJ} / \mathrm{mol}$. Por su parte el factor pre-exponencial de la ecuación de Arrhenius (Do) obtuvo valores de $4.123 \times 10^{-6} \mathrm{~m}^{2} / \mathrm{s}$ (intervalo de confianza 95\%: -1.483 x 10-6; $9.729 \times 10^{-6}$ ) y $2.463 \times 10^{-6} \mathrm{~m}^{2} / \mathrm{s}$ (intervalo de confianza $95 \%$ : $-1.001 \times 10^{-5} ; 1.493 \times 10^{-5}$ ) para la variedad Obonuco Andina y Sureña, respectivamente.

En las ecuaciones 6 y 7 se presentan las ecuaciones que relacionan el coeficiente de difusividad efectiva $\left(\mathrm{m}^{2} / \mathrm{s}\right)$ y la temperatura de secado $\left({ }^{\circ} \mathrm{C}\right)$ :

Para variedad Obonuco Andina:

$\mathrm{D}_{\text {eff }}=\left(4.123 \times 10^{-6} \frac{\mathrm{m}^{2}}{\mathrm{~s}}\right) \exp \left(-\frac{26.867 \frac{\mathrm{kJ}}{\mathrm{mol}}}{\mathrm{R}(\mathrm{T}+273.15)}\right) \quad\left(\mathrm{R}^{2}{ }_{a d j}=0.998\right)$

Para variedad Sureña: 
$\mathrm{D}_{\mathrm{eff}}=\left(2.463 \times 10^{-6} \frac{\mathrm{m}^{2}}{\mathrm{~s}}\right) \exp \left(-\frac{25.291 \frac{\mathrm{kJ}}{\mathrm{mol}}}{\mathrm{R}(\mathrm{T}+273.15)}\right) \quad\left(\mathrm{R}_{a d j}^{2}=0.975\right)$

Existe variación en los parámetros calculados (difusividad efectiva, al igual que en la energía de activación y factor pre-exponencial de la ecuación de Arrhenius) entre las variedades de arveja estudiadas. Sin embargo los valores de $\mathrm{R}^{2}$ adj de las ecuaciones 6 y 7 indican que una ecuación tipo Arrhenius con parámetros Ea y $\mathrm{D}_{0}$ constantes explica más del $97 \%$ de la variación de los datos experimentales. Esto permite suponer que los parámetros podrían ser considerados constantes para el rango de condiciones estudiadas. Por otro lado existen diferencias de los resultados reportados en investigaciones citadas, esto puede atribuirse principalmente a diferencias en la técnica de secado, el equipo y las condiciones de temperatura y humedad relativa.

\section{CONCLUSIONES}

Las variedades estudiadas tuvieron un comportamiento cinético similar, los resultados obtenidos para la pérdida de humedad con respecto al tiempo de secado a 45, 50, 55 y $60^{\circ} \mathrm{C}$ denotaron curvas similares a las reportadas por otros estudios en arveja, de igual forma evidencian la reducción del tiempo de secado con el incremento de temperatura.

Los datos de cinética de secado expresados como razón de humedad con respecto al tiempo, fueron ajustados a ocho modelos reportados por literatura y mostraron buenos resultados de ajuste. Sin embargo, a partir los parámetros estadísticos estudiados, el modelo Verma es el que mejor representa la cinética de secado para las variedades de arveja Obonuco Andina y Sureña.

Por medio de la energía de activación y el factor pre-exponencial se obtuvo una ecuación tipo Arrhenius que describió el efecto de la temperatura sobre el coeficiente de difusividad efectiva. El orden de magnitud de los resultados obtenidos corresponde a los reportados por literatura para otros productos agroalimentarios.

\section{AGRADECIMIENTOS}

Al personal y la planta docente de la Universidad de Nariño, al Sistema General de Regalías por el aporte de los recursos en el proyecto BPIN 2012000100188 con el cual se realizaron parte de los estudios. Al grupo de investigación Cedagritol de la Universidad del Tolima por contribuir a esta investigación por medio de sus investigadores e instalaciones.

\section{REFERENCIAS}

Barroca, M. J., y Guiné, R. P. F., Drying Kinetics of Banana Slices (cvs. Musa nana and Musa Cavendish), http://repositorio.ipv.pt/handle/10400.19/1765; ISSN 2010-3778, WASET, (79), 633-636. (2013)

Bezerra, C. V., Meller da Silva, L. H., Corrêa, D. F., Rodrigues, A. M. C., A modeling study for moisture diffusivities and moisture transfer coefficients in drying of passion fruit peel, doi: 10.1016/j.jjheatmasstransfer.2015.02.027, Int. J. Heat Mass Tran, 85, 750-755 (2015)

Bispo, J. a. C., Bonafe, C. F. S., Santana, K. M. O. V., Santos, E. C., A comparison of drying kinetics based on the degree of hydration and moisture ratio, doi: 10.1016/j.Iwt.2014.07.014, Food Sci. Technol. Int., 60(1), 192-198 (2015)

Chinenye, N. M., Ogunlowo, A., Olukunle, O., Cocoa bean (Theobroma cacao L.) drying kinetics, doi: 10.4067/S0718-58392010000400014, Chil. J. Agr. Res., 70(4), 633-639 (2010)

Corrêa, P.., Mendes, F., Horta, G., Duarte, A., Resende, O., Campos, S., Mathematical modeling of the drying process of corn ears, doi: 10.4025/actasciagron.v33i4.7079, Acta Sci., Agron., 33(4), 575-581 (2011)

Corzo, O., Bracho, N., Alvarez, C., Weibull model for thin-layer drying of mango slices at different maturity stages, doi: 10.1111/j.1745-4549.2009.00433.x, J Food Process Preserv, 34(6), 993-1008 (2010)

Djendoubi, N., Boudhrioua, N., Kechaou, N., Courtois, F., Bonazzi, C., Influence of air drying temperature on kinetics, physicochemical properties, total phenolic content and ascorbic acid of pears, doi: :10.1016/j.fbp.2011.11.009, Food Brioprod. Process, , 90(3), 433-441 (2012)

Doymaz, I, Drying behaviour of green beans, doi: 10.1016/j.jfoodeng.2004.08.009, J. Food Eng., 69(2), 161$165(2005)$ 
Doymaz, I., y Kocayigit, F., Drying and Rehydration Behaviors of Convection Drying of Green Peas, doi: 10.1080/07373937.2011.591713, Drying Technol., 29(11), 1273-1282 (2011)

Dutta, P. P., y Baruah, D. C., Drying modelling and experimentation of Assam black tea (Camellia sinensis) with producer gas as a fuel, doi: 10.1016/j.applthermaleng.2013.11.035, App. Therm. Eng., 63(2), 495-502 (2014)

Eim, V. Optimización del proceso de secado en base a criterios de calidad. Aplicación al diseño de un alimento cánico enriquecido en fibra alimentaria, Tesis doctoral, Dpto de Química, Univ de las Illes Balears, Palma de Mallorca - Islas Baleares (2012)

Fonseca, F., Castro Melo, E., S. Santos, R. H., Pereira da Rocha, R., Martinazzo, A. P., Radunz, L. L., Navarro García, M., Evaluation of mathematical models for prediction of thin-layer drying of brazilian lemonscented verbena leaves (Lippia alba (MILL) N.E. BROWN), http://www.deag.ufcg.edu.br/rbpa/rev91/Art919.pdf; ISSN 1517-8595, Rev. Bras. Prod. Agroind., 9(1), 71$80(2007)$

García, M., Alvis, A., García, C., Evaluación de los pretratamientos de la deshidratación osmótica y microondas en la obtención de hojuelas de mango (Tommy Atkins), doi: 10.4067/S071807642015000500009 Inf. Tecnol, 26(5), 63-70 (2015)

Garg, M., Sharma, S., Varmani, S., Sadhu, S., Drying kinetics of thin layer pea pods using tray drying, http://www.ijfans.com/currentissue.html, ISSN: 2320-7876, IJFANS, 3(3), 61-66 (2014)

Guiné, R. P. F., Barroca, M. J., Effect of Drying on the Textural Attributes of Bell Pepper and Pumpkin, doi: 10.1080/07373937.2011.596297, Drying Technol., 29(16), 1911-1919 (2011)

Hacihafizoğlu, O., Cihan, A., Kahveci, K., Mathematical modelling of drying of thin layer rough rice, doi :10.1016/j.fbp.2008.01.002, Food Bioprod. Process,, 86(4), 268-275 (2008)

Hatamipour, M. S., y Mowla, D., Correlations for shrinkage, density and diffusivity for drying of maize and green peas in a fluidized bed with energy carrier, doi: 10.1016/S0260-8774(02)00461-2, J. Food Eng., 59(23), 221-227 (2003)

Hernández, C., Ossa, Z., Ramírez, L., y Herrera, W., Influencia del espesor y la temperatura en el secado de carambola (averrhoa carambola L.), http://www.udla.edu.co/revistas/index.php/ingenierias-yamazonia/article/view/91/131-142, ISSN: 1692-7389, I. \& y A., 4(2), 131-142 (2011)

Hui, Y., Modeling, Handbook of food science, technology and engineering, 3er Volumen, pp 13-14, Taylor \& Francis, New York, USA (2006)

Jovanovic, S. Quality Characterization and Modeling Experimental Kinetics in Pilot Scale Heat Pump Drying of Green Peas, Tesis de Magister, Depto. Energía y Procesos Ingenieriles, Universidad Noruega de Ciencia y Tecnología, Trondheim, Noruega (2013).

Joykumar, N. y Pandey, R., Convective air drying characteristics of sweet potato cube (Ipomoea batatas L.), doi: 10.1016/j.fbp.2011.06.006, Food Bioprod. Process., 90(2), 317-322 (2012)

Kaleta, A., y Górnicki, K., Evaluation of drying models of apple (var. Ligol) dried in a fluidized bed dryer, doi: 10.1111/j.1365-2621.2010.02230.x, Int. J. Food. Sci. Tech., 45, 891-898 (2010)

Kaymak, F. Drying and Rehydrating Kinetics of Green and Red Peppers, doi: 1 0.1111/j.13652621.2002.tb11378.x, JFS, 67(1), 168-175 (2002)

Machado, A., Oliveira, E., Santos, E., Oliveira, J. Estudio del secado de anacardo (Anacardium occidentale L.) mediante secador solar de radiación directa, doi: 10.1612/inf.tecnol.4137it.08, Inf. Tecnol., 21(1), 31-37 (2010)

Martinez, G., Flores, A., Mercado, J., y López, M. Características de secado de nopal (Opuntia ficus-indica) por lecho fluidizado, http://www.actauniversitaria.ugto.mx/index.php/acta/article/viewFile/61/48, ISSN: 01886266, Acta Univ., 20(3), 70-76 (2010)

Martínez, C., Influencia de las condiciones de secado en las cinéticas de deshidratación de pepitas de uva, Tesis de maestría, Departamento de Tecnología de Alimentos, Universidad Politécnica de Valencia, Valencia, España (2012) 
Mechlouch, R. F., Mahdhaoui, B., Elfalleh, W., Mahjoubi, A., Mathematical Modeling of Microwave Drying of Beans (Vicia faba L), Peas (Pisum sativum) and Tomatoes (Rio grande) in Thin Layer, doi: 10.5923/j.ijee.201401.04, IJEE, 4(2), 25-32 (2014)

Menges, H., y Ertekin, C., Mathematical modeling of thin layer drying of Golden apples, doi: 10.1016/j.jfoodeng.2005.06.049, J. Food Eng., 77(1), 119-125 (2006)

Michalewicz, J., Henriquez, J., Charamba, J., Secado de Cajuil (anacardium occidentale I.): Estudio experimental y modelado de la cinética de secado, doi: 10.4067/S0718-07642011000600007, Inf. Tecnol., 22 (6), 63-74 (2011)

Mujumdar, A., Basic Process Calculations and Simulation in Drying, Hadnbook of Industrial Drying, $3^{\text {ra }}$ edición, 54 - 79. Taylor \& Francis Group, New York, USA (2007)

Pardeshi, I. L., Arora, S., Borker, P., Thin-Layer Drying of Green Peas and Selection of a Suitable Thin-Layer Drying Model, doi: 10.1080/07373930802606451, Drying Technol., 27(2), 288-295 (2009)

Perea, M. de J., Efecto del secado por lecho fluidizado en la estructura de semillas de Ricinus communis y en la extracción de su aceite como fuente alternativa de biocombustibles, Tesis doctoral, Escuela Nacional de Ciencias Biológicas, Instituto Politécnico Nacional, Ciudad de México, México (2011)

Priyadarshini, Shukla, R. N., Mishra, A. A., Microwave Drying Characteristics of Green Peas and its Quality Evaluation, http://www.ripublication.com/ijafst_spl/ijafstv4n5spl_08.pdf, ISSN: 2249-3050, IJAFST, 4(5), 445-452 (2013)

Puente, L., Echegaraay, E., Castro, E., y Di Scala, K., Secado asistido por infrarrojos de descartes de limón (Citrus Limon ( L.) Burm . F . Cv . Genova ), http://www.bdigital.unal.edu.co/34276/1/34404-181698-1PB.pdf, ISSN: 0012-7353, Dyna, 80(181), 91-97 (2013)

Rafiee, S., Keyhani, A., Sharifi, M., Jafari, A., Mobli, H., Tabatabaeefar, A., Thin Layer Drying Properties of Soybean (Viliamz Cultivar), http://jast.modares.ac.ir/article_4309_921.html, ISSN: 2345-3737, J. Agric. Sci. Technol, 11, 289-300 (2009)

Rodriguez, J., Contribución al Estudio de la Intensificación del Proceso de Secado de Tomillo (Thymus Vulgaris L.): Aplicación de Ultrasonidos de Potencia y Secado Intermitente, Tesis doctoral, Depto de Tecnología de Alimentos, Universidad Politécnica de Valencia, Valencia, España (2013)

Sahin, S., Sumnu, G., Tunahoyu, F., Usage of solar-assisted spouted bed drier in drying of pea, doi: 10.1016/j.fbp.2012.11.006, Food Bioprod. Process., 91, 271-278 (2013)

Senadeera, W., The Drying Constant and its Effect on the Shrinkage Constant of Different-Shaped Food Particulates, http://eprints.qut.edu.au/16993/1/16993.pdf, ISSN: 2301-3664, Int. J. Food Eng., 4(8), 1 - 16 (2008)

Simal, S., Mulet, A., Tarrazo, J., Rosselló, C., Drying models for green peas, doi: 10.1016/03088146(95)00074-7, Food Chem. 55(2), 121-128 (1996)

Thuwapanichayanan, R., Prachayawarakorn, S., Kunwisawa, J., Soponronnarit, S., Determination of effective moisture diffusivity and assessment of quality attributes of banana slices during drying, doi: :10.1016/j.Iwt.2011.01.003, LWT - Food Sci. Technol., 44(6), 1502-1510. (2011)

Torregroza, A. M., Montes, E. J., y Ávila, A. E., Modelado de las cinéticas de secado de tres variedades de yuca industrial, http://dx.doi.org/10.15446/dyna.v81n186.39923, ISSN: 0012-7353, Dyna, 81(186), 184-192 (2014)

Torrez, R., y Giner, S. Drying-toasting kinetics of presoaked soybean in fluidised bed. Experimental study and mathematical modelling with analytical solutions, doi: 10.1016/j.jfoodeng.2013.12.009, J. Food Eng., 128, 31-39 (2014)

Váquiro, H. Contribución al estudio y optimización del secado intermitente : aplicación al secado de mango ( Mangifera indica L var. Tommy Atkins), Tesis doctoral, Depto de Tecnología de Alimentos, Universidad Politécnica de Valencia, Valencia, España (2009)

Vega, A., Andrés, A., Fito, P. Modelado de la cinética de secado del pimiento rojo (Capsicum annum L. cV Lamuyo), http://dx.doi.org/10.4067/S0718-07642005000600002, ISSN: 0718-0764, Inf. Tecnol., 16(6), 3-11 (2005) 
Villa-Velez, H., Váquiro, H. y Telis, J., The effect of power-ultrasound on the pretreatment of acidified aqueous solutions of banana flower-stalk: Structural, chemical and statistical analysis, doi: 10.1016/j.indcrop.2014.12.022, Ind. Crops Prod., 66, 52 - 61 (2015)

Zielinska, M., Zapotoczny, P., Alves-Filho, O., Eikevik, T. M., Blaszczak, W., A multi-stage combined heat pump and microwave vacuum drying of green peas, doi: 10.1016/j.jfoodeng.2012.10.047, J. Food Eng., 115(3), 347-356 (2013) 